\section{Compact desktop milling solutions}

DGSHAPE Corporation, a leading provider of dental milling solutions, has announced the launch of two new 5-axis dental mills: the DWX-52DCi Automatic Disc Changer model, with new DWINDEX software for monitoring return-on-investment and productivity at a glance, and the DWX-52D, the successor model to the industry-standard DWX-51D.

Since their introduction in 2010, DWX-series dental mills have gained widespread recognition and industry awards for their compact desktop size, user-friendliness, robust reliability and open architecture, which has contributed to the mainstream digitalisation of dental laboratories worldwide. The DWX-52DCi and DWX-52D add exciting new features and capabilities to further automate and optimise the digital dental workflow.

The DWX-52DCi features an Automatic Disc Changer (ADC), capable of loading up to six discs of different materials, enabling discs to be replaced automatically during milling to maximise lab productivity. In addition, the DWX-52DCi supports new DWINDEX management software for monitoring key productivity indexes, including operation time, materials used and deliverables.

The DWX-52D is equipped with a new snap-on clamp system which offers the fast and secure set-up of various materials and maximises disc usage, making lab operation easier and more efficient than ever. Both mills feature cutting-edge dental technologies, including an Automatic Tool Changer (ATC) for fully automated 5-axis milling of a wide range of materials, including Zirconia, wax, PMMA, gypsum, PEEK, glass fibre-reinforced resin, composite resin and $\mathrm{CoCr}$ sinter metal. Typical applications include crowns, copings, bridges, inlays and onlays, veneers, abutments, implant bars, screw-retained crowns, dentures, models, surgical guides and bite splints.

The ATC is capable of holding 15 tools for uninterrupted milling overnight, with an additional slot dedicated to a cleaning tool for maintaining a clean milling operation. Continuing DGSHAPE's reputation for high-quality, precision milling, both mills include a rigid frame with ball screw axis control, automatic calibration of all five axes, and an ioniser for reducing static electricity when milling PMMA. Air pressure is adjusted automatically, depending on the type of material being milled, for continuous, reliable production.

Other advanced features include a Virtual Machine Panel (VPanel) which allows users to quickly configure settings for up to four machines from a single computer for high-volume production. To help minimise downtime, the VPanel monitors operational status and sends email notifications at the end of milling or if operator intervention is required.

DGSHAPE is the new name for Roland Easy Shape.

To discover more visit www.rolanddg.co.uk.

\section{Make marginal gains in decontamination}

Dentistry is all about marginal gains - how can each aspect of practice be improved to achieve the most efficient and effective outcome. With decontamination the answer can be found with the new Assistina TWIN from leading manufacturer, $\mathrm{W} \& \mathrm{H}$.

As the first reprocessing device to be equipped with two process chambers that can be used alternately for non-stop loading, the Assistina TWIN is extremely quick and powerful to use. It takes just ten seconds to reprocess each handpiece making it possible to process up to 360 handpieces per hour to a consistently high standard. Now, results are guaranteed twice as fast - what more could you ask for?

Actually, there's a lot more you could ask for, and with the Assistina TWIN you get it all from innovative oil nebulisation technology to a cost effective design so that each refill maintains approximately 2,800 instruments. Together with the unit's intuitive and easy to use control panel, you're sure to make marginal gains in decontamination in your practice.

To find out more visit www.wh.com/ en_uk, call 01727874990 or email office. uk@wh.com.

\title{
Early oral cancer detection
}

How confident are you in detecting an early cancerous lesion? Sadly, oral cancer is often caught in the late stages, which is why mortality rates are higher. If caught early, the survival rate is over $85 \%$ when cancers are small.

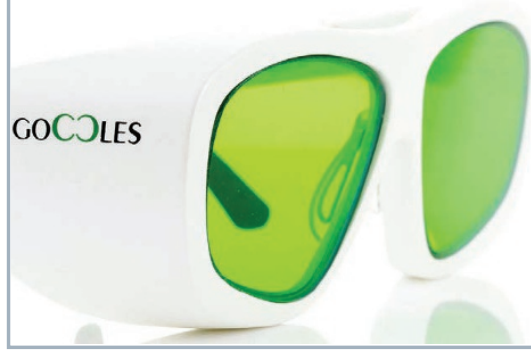

As well as visual screening, practices are also using technology to assist in diagnosing early cancerous lesions. One such device is Goccles, Oral Cancer Screening Glasses. These allow the clinician to run a simple, noninvasive and painless test using the technology of fluorescence and cell-tissue autofluorescence by utilising the wavelengths emitted by curing-lights. This allows the user to see in a clear and accurate way any anomalies of the oral cavity. The basic principle is that the auto-fluorescence of abnormal cells lining the mouth when exposed to light differs to that seen occurring in normal cells. Goccles glasses allow the clinician to see differences in autofluoresence of the tissues, with normal cells appearing green and abnormal cells dark.

The eyewear is comfortable to wear and permits the simultaneous wearing of standard glasses. The light source is held by an assistant, leaving the examining clinician with both hands free to reflect the oral soft tissues with dental mirrors to ensure that the entire oral mucosa is assessed.

Goccles are distributed exclusively through Dental Sky. For more information visit https://www.dentalsky.com/gocclesoral-cancer-screening-glasses.html. 